\title{
Aplicación de la ecuación de Schrodinger en heteroestructuras semiconductoras de baja dimensionalidad
}

\author{
Aplication of Schrodinger equation in low-dimensionality \\ semiconducting heterostructures
}

\author{
Francis Armando Segovia \\ Físico, Magíster en Ciencias-Física. Docente de la Universidad Distrital Francisco Jose \\ de Caldas. Bogotá, Colombia. Contacto: fasegoviac@udistrital.edu.co \\ Fecha de recepción: 1 de abril de 2012 \\ Clasificación del artículo: Investigación \\ Fecha de aceptación: 12 de febrero de 2013 \\ Financiamiento: Universidad Distrital Francisco Jose de Caldas
}

Palabras clave: ecuación de Schrodinger, energía del estado fundamental, presión hidrostática.

Key words: Schrodinger equation, energy level of the ground state, hydrostatic pressure.

\section{RESUMEN}

La investigación presentada en este artículo está orientada hacia el área de matería condensada en el campo de la física de semiconductores. En esta investigación se utilizan los principios básicos de la mecánica cuántica, en especial el de la aproximación de masa efectiva. El objetivo de este artículo es determinar las energías del estado fundamental, así como las energías de transición electrón-hueco cuando una heteroestructura semiconductora de $G a A s-G a_{1-x} A l_{x} A s$ está inmersa en una barrera de $G a_{1-}$ $A l_{y} A s$, por la aplicación de una presión hidrostática. La metodología que se propone en el presente trabajo es solucionar analíticamente la ecuación diferencial de segundo orden de Schrodinger. Las soluciones encontradas permiten determinar las funciones propias de la ecuación diferencial y además las energias de transicion en el estado fundamental mediante la aplicación de la presión hidrostática. Los principales resultados fueron encontrados mediante el uso del software Mathematica 5.0, mostrando que en el regimen de confinamiento fuerte, para pequeños anchos del pozo de potencial de la heteroestructura semiconductora, el potencial de confinamiento con la presion es menor para la función de los portadores de carga (electrón-hueco). Sin embargo, se muestra que en el regimen de confinamiento débil los efectos de la presión hidrostática sobre las alturas de la barrera son más significativos, y las energías de los portadores disminuyen.

\section{ABSTRACT}

The research presented in the following paper concerns condensed matter areas in the field of semiconductor physics. This research uses the basic principles of quantum mechanics, particularly effective mass approximation. The aim of this paper is to determine the energies of ground state as well as the energies of electron-hole transition when a semiconductor heterostructure GaAs-Ga1-xAlxAs is 
immersed in a barrier of Ga1-yAlyAs, by applying hydrostatic pressure. The methodology proposed in the present work analytically solves Schrödinger's second-order differential equation to find solutions and allow determining the corresponding differential equation together with the energies of transition in the ground state. This is accomplished through the application of hydrostatic pressure. The main results were obtained using software package
Mathematica 5.0. Results indicate a regime of strong confinement for small widths of the potential well in semiconductor heterostructures, where the confinement potential lessens with pressure for the charge-carrier function (electron-hole). However, the findings demonstrate that, in the regime of weak confinement, the effects of hydrostatic pressure on the heights of the barrier are more significant, and there is also a reduction in carrier energies.

\section{INTRODUCCIÓN}

Aunque el uso de materiales semiconductores para aplicaciones electrónicas se remonta a mediados del siglo anterior, no fue sino hasta principios de los años setenta, con el trabajo pionero de Esaki y Tsu [1], cuando se sugirió la idea de construir estructuras artificiales de estos materiales. En el contexto del desarrollo de la tecnología de los semiconductores, las heteroestructuras surgieron con la finalidad de diseñar dispositivos en los que fuese posible tener un cierto control sobre sus propiedades ópticas y electrónicas, es decir, en cierto modo, poder diseñar dispositivos electrónicos a la carta. En una primera etapa de desarrollo de las heteroestructuras de semiconductores, los éxitos obtenidos no fueron muy numerosos debido, en parte, a la falta de control sobre las imperfecciones inherentes a los procesos de crecimiento de los distintos materiales. Los primeros éxitos vinieron con la fabricación de láseres de inyección a partir de heterouniones de GaAs-AlGaAs, que fueron crecidas mediante la técnica de epitaxia en fase líquida (LPE) [2], [3].

No en vano, el desarrollo de las técnicas de crecimiento cristalino epitaxial, que permitió la descomposición secuencial de capas atómicas sobre la superficie de un cristal, supuso el nacimiento de la nanoelectrónica. Su nacimiento ha originado una auténtica revolución tecnológica, vigente en la actualidad, que se encamina a la miniaturización; es decir, hacia la integración de un gran número de componentes (transistores, condensadores y resistencias) en unas pequeñas dimensiones y hacia la fabricación de nuevos materiales con propiedades fotoluminisentes, de conducción eléctrica, etc. [4]. Sus propiedades optoelectrónicas dependen de los parámetros de diseño seleccionados (propiedades químicas de los materiales que se combinan y grosor de las capas de los materiales escogidos) por lo que, previamente a la síntesis, es posible diseñar de forma teórica la nanoestructura conforme a las particulares aplicaciones con las que se conciba.

Todo el interés por construir heteroestructuras reside en el hecho de que, a pesar de lo complicado del proceso de crecimiento, es posible manipular el comportamiento de electrones y huecos mediante lo que se ha dado en llamar ingeniería de bandas. Con el desarrollo de las distintas técnicas epitaxiales de crecimiento de superficies a mediados de los setenta, en particular de la deposición química de vapores (CVD) y de la epitaxia por haces moleculares (MBE), comenzó a surgir una nueva tecnología basada en el diseño de diferentes tipos de heteroestructuras artificiales. Diodos emisores de luz, transistores bipolares, transistores de efecto campo, láseres de cascada, etc., han ido apareciendo, y sus propiedades se han visto mejoradas merced al empuje realizado, tanto desde el punto de vista tecnológico como desde el punto de vista de la mejor comprensión teórica de la física de estos sistemas. 


\section{|investigación}

Sin embargo, desde un punto de vista aplicado, las heteroestructuras de semiconductores constituyen una excelente herramienta para estudiar los más diversos fenómenos físicos ante la posibilidad de jugar con su dimensionalidad a la hora de confinar los portadores de carga en cero, una y dos dimensiones. Así, se han usado superredes, pozos cuánticos, barreras dobles, etc., para investigar, desde un punto de vista fundamental, diversos fenómenos como el efecto Hall cuántico, la localización en sistemas desordenados y la transición metal-aislante [5], [6].

Durante mucho tiempo, la investigación en el campo de los sistemas electrónicos se limitó al estudio de los átomos, moléculas y cristales que nos proporciona la naturaleza. Estos sistemas son tridimensionales, cuando el confinamiento espacial es del mismo orden en las tres direcciones del espacio, aunque también existen ordenamientos cuasi-unidimensionales o cuasi-bidimensionales, sobre todo de substancias poliméricas. Sin embargo, la flexibilidad y control en el diseño de estructuras semiconductoras de tamaño nanoscópico en las cuales los portadores de carga se confinan a voluntad en una, dos o tres direcciones del espacio, supuso una auténtica innovación tanto desde el punto de vista de las aplicaciones como de la investigación básica. Los puntos cuánticos $Q D$ 's $(O D)$ son un miembro de las estructuras cuánticas de baja dimensión, entre las que también se encuentran los pozos cuánticos $Q W^{\prime} s(2 D)$ y los hilos cuánticos $Q W W^{\prime} s(1 D)$. La diferencia entre estas estructuras reside en el grado de confinamiento. Un pozo cuántico se sintetiza por la deposición de unas capas atómicas de material $B$, que se sitúan en un cristal de material $A$, el cual presenta una energía para la banda de conducción superior a la de material $B$.

En el presente trabajo se analiza el estudio de las energías del estado fundamental de portadores (electrón/hueco) en función de la geometría del sistema a estudiar y por otro lado, se tiene en cuenta el efecto de la presión hidrostática sobre los estados de energía de portadores de carga y de transición electrón-hueco.

\section{METODOLOGÍA}

El sistema objeto de estudio consiste en una caja cuántica de GaAs rodeada de $G a_{1-x} A l_{x} A s$. En la figura 1 se muestran las capas de los diferentes materiales utilizados y los esquemas de energías correspondientes. Consideramos la dirección [100] como la dirección de crecimiento de la estructura:
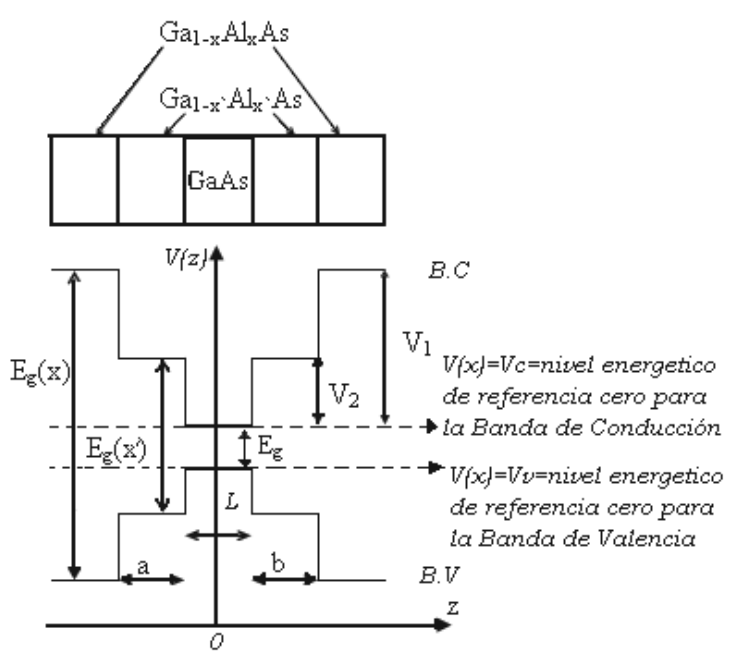

Figura 1. Representación de la heteroestructura semiconductora. Fuente: elaboración propia.

El Hamiltoniano de un electrón (hueco) en ausencia de campo magnético, es determinado por el potencial de confinamiento de la capa de $G a_{1-x} A l_{x} A s$ que está rodeada por la estructura de $G a_{1-y} A l_{y} A s$ :

$$
H=\frac{p_{e, h}^{2}}{2 m_{e, h}^{*}}+V_{e, h}(z, \rho)
$$

donde el primer término corresponde a la energía cinética del electrón (hueco), $m_{e}^{*}\left(m_{h}^{*}\right)$ es la correspondiente masa efectiva y el segundo término es el potencial de confinamiento dado por:

$$
V(z)=\left\{\begin{array}{lll}
0 & \text { para } & -L / 2 \leq z \leq L / 2 \\
V_{1} & \text { para } & z<-a-L / 2 \quad, z>L / 2+b \\
V_{2} & \text { para } & -L / 2>z \geq-a-L / 2, \quad L / 2<z \leq L / 2+b
\end{array}\right.
$$




\section{investigación |}

$$
V(\rho)=\left\{\begin{array}{lll}
0 & \text { para } & \rho<R \\
V_{2} & \text { para } & \rho \geq R
\end{array}\right.
$$

A continuación vamos a solucionar el Hamiltoniano ecuación (1) para el sistema ilustrado en la figura 1 , por simplicidad vamos a obviar los subíndices. Para la solución del hamiltoniano del problema considerado, la ecuación de Schrodinger estacionaria viene dada por:

$$
H \Psi(\rho, \varphi, z)=\left[-\frac{\hbar^{2}}{2 m^{*}} \nabla^{2}+V(\rho, z)\right] \Psi(\rho, \varphi, z)=E \Psi(\rho, \varphi, z)
$$

Debido a la simetría, en coordenadas cilíndricas ecuación (4) se reescribe de la siguiente forma,

$$
-\frac{\hbar^{2}}{2 m^{*}}\left[\frac{1}{\rho} \frac{\partial}{\partial \rho}\left(\rho \frac{\partial \Psi}{\partial \rho}\right)+\frac{1}{\rho^{2}} \frac{\partial^{2} \Psi}{\partial \varphi^{2}}+\frac{\partial^{2} \psi}{\partial z^{2}}\right]+V(\rho, z) \Psi=E \Psi
$$

De acuerdo al método de separación de variables, podemos escribir la función de onda como:

$$
\Psi(\rho, \varphi, z)=\psi(z) \chi(\rho) \phi(\varphi)
$$

Reemplazando ecuación (6) en la ecuación (5), obtenemos las ecuaciones diferenciales que rigen la dependencia de cada una de las funciones de la ecuación (6) y se obtiene la ecuación (7), (8) y (9),

$$
\frac{1}{\rho} \frac{\partial}{\partial \rho}\left(\rho \frac{\partial \chi}{\partial \rho}\right)-\frac{2 m^{*}}{\hbar^{2}}\left[V(\rho)+\frac{m^{\prime 2}}{\rho^{2}}-E_{\rho}\right] \chi=0
$$

$$
-\frac{\hbar^{2}}{2 m^{*}} \frac{\partial^{2} \psi}{\partial z^{2}}+\left[V(z)-E_{z}\right] \psi=0
$$

$$
\frac{\partial^{2} \phi}{\partial \varphi^{2}}+\frac{2 m^{*} m^{\prime}}{\hbar^{2}} \phi=0
$$

En las ecuaciones anteriores, $E_{\rho}$ y $E_{z}$ representan respectivamente las energías en la parte radial $\rho$ yen la dirección $z$ de crecimiento de la estructura, para ello debemos tener las condiciones dadas por la ecuación (2) y la ecuación (3). Además, definimos a $m^{\prime}$ como una constante de separación, para el caso de interés trabajaremos en el estado fundamental donde $m^{\prime}=0$, por lo tanto las soluciones de la ecuación (9) serán de la forma $\phi(\varphi)=e^{i m^{\prime} \varphi}=1$ . Fácilmente se encuentra que las soluciones de las ecuaciones diferenciales ecuación (7) y ecuación (8) vienen dadas por:

$$
\chi(\rho)=\left\{\begin{array}{lll}
A J_{0}\left(k^{\prime} \rho\right) & \text { para } & \rho \prec R \\
B K_{0}(k \rho) & \text { para } & \rho \geq R
\end{array}\right.
$$

$\psi(z)=\left\{\begin{array}{lll}C e^{i k_{1} z}+D e^{-i k_{1} z} & \text { para } & -L / 2<z<L / 2 \\ F e^{k_{2} z}+G e^{-k_{2} z} & \text { para } & -a-L / 2 \leq z \leq-L / 2 \\ H e^{k_{2} z}+M e^{-k_{2} z} & \text { para } & L / 2 \leq z \leq L / 2+b \\ N e^{k_{3} z} & \text { para } & z \leq-a-L / 2 \\ L e^{-k_{3} z} & \text { para } & z \geq L / 2+b\end{array}\right.$

Siendo $J_{0}\left(k^{\prime} \rho\right)$ y $K_{0}(k \rho)$ las funciones de Bessel de primera y segunda especie respectivamente, $A, B, C, D, F, G, H, M, N$ y $L$ constantes, mientras que $k_{1}, k_{2}, k_{3}, k^{\prime}$ y $k$ vienen determinadas por la ecuación (12):

$$
\begin{gathered}
k_{1}=\sqrt{\frac{2 m^{*}}{\hbar^{2}} E_{z}}, \quad k_{2}=\sqrt{\frac{2 m^{*}}{\hbar^{2}}\left(V_{1}-E_{z}\right)}, \quad k_{3}=\sqrt{\frac{2 m^{*}}{\hbar^{2}}\left(V_{2}-E_{z}\right)}, \\
k^{\prime}=\sqrt{\frac{2 m^{*}}{\hbar^{2}} E_{\rho}} \quad y \quad k=\sqrt{\frac{2 m^{*}}{\hbar^{2}}\left(E_{\rho}-V_{2}\right)}
\end{gathered}
$$


Las anteriores funciones de onda nos permitirán determinar la energía del estado base paraelectrón (hueco) en el sistema considerado.

A continuación presentamos los resultados que se obtienen para las energías del estado fundamental del electrón (hueco), para ello se aplican las condiciones usuales de continuidad para las funciones de onda y sus derivadas en cada una de las fronteras (figura 1). Los resultados numéricos presentados en la siguiente sección se obtienen mediante el uso del paquete matemático Mathematica 5.0.

Las energías en el estado fundamental de los electrones (huecos) se determinan a partir de la solución de las ecuaciones trascendentales en $\rho$ y $z$, que se consiguen de la ecuación (10) y la ecuación (11), al aplicar las condiciones de frontera sobre cada una de las funciones de onda, se demuestra que las ecuaciones trascendentales a resolver son de la forma:

$$
k J_{0}\left(k^{\prime} R\right)=k^{\prime} \frac{J_{1}\left(k^{\prime} R\right)}{K_{1}(k R)} K_{0}(k R)
$$

$Z_{X}\left(k_{2}^{2}-k_{2} \gamma-k_{1}^{2}\right) \cos \left(\frac{k_{1} b}{2}\right)-Z_{X}\left(2 k_{1} k_{2}-k_{1} \gamma\right) \sin \left(\frac{k_{1} b}{2}\right)-Z_{Y} k_{1} \gamma \sin \left(\frac{k_{1} b}{2}\right)+$

$Z_{X}\left(2 k_{1} k_{2}-k_{1} \gamma\right) \sin \left(\frac{3 k_{1} b}{2}\right)+Z_{Y}\left(k_{2}^{2}-k_{2} \gamma+k_{1}^{2}\right) \cos \left(\frac{k_{1} b}{2}\right)+Z_{Y} k_{1} \gamma \sin \left(\frac{3 k_{1} b}{2}\right)$

$Z_{Y}\left(k_{2} \gamma-k_{2}^{2}+k_{1}^{2}\right) \cos \left(\frac{3 k_{1} b}{2}\right)+Z_{X}\left(k_{2} \gamma-k_{2}^{2}+k_{1}^{2}\right) \cos \left(\frac{3 k_{1} b}{2}\right)=0$.

En la ecuación (14) hemos utilizado las siguientes substituciones, dadas por ecuación (15),

$$
\begin{gathered}
Z_{X}=\left(k_{3}+k_{2}\right) e^{k_{2} d}, Z_{Y}=\left(k_{3}-k_{2}\right) e^{-k_{2} d} \mathrm{y} \\
\gamma=\frac{2 k_{2}\left(k_{2}-k_{3}\right)}{\left(k_{2}+k_{3}\right) e^{2 k_{2} a}+\left(k_{2}-k_{3}\right)}
\end{gathered}
$$

En definitiva, el problema planteado en este trabajo se resuelve mediante las soluciones de la ecuación (13) y la ecuación (14).

\section{RESULTADOS}

En la figura 2 presentamos la energía del estado base en un punto cuántico de GaAs con radio de $100 \AA$ Å como función de la longitud del punto para diferentes espesores de las barreras de potencial. La figura 2(a) representa la energía del estado base para electrones, mientras que para los huecos viene representado por la figura 2 (b).
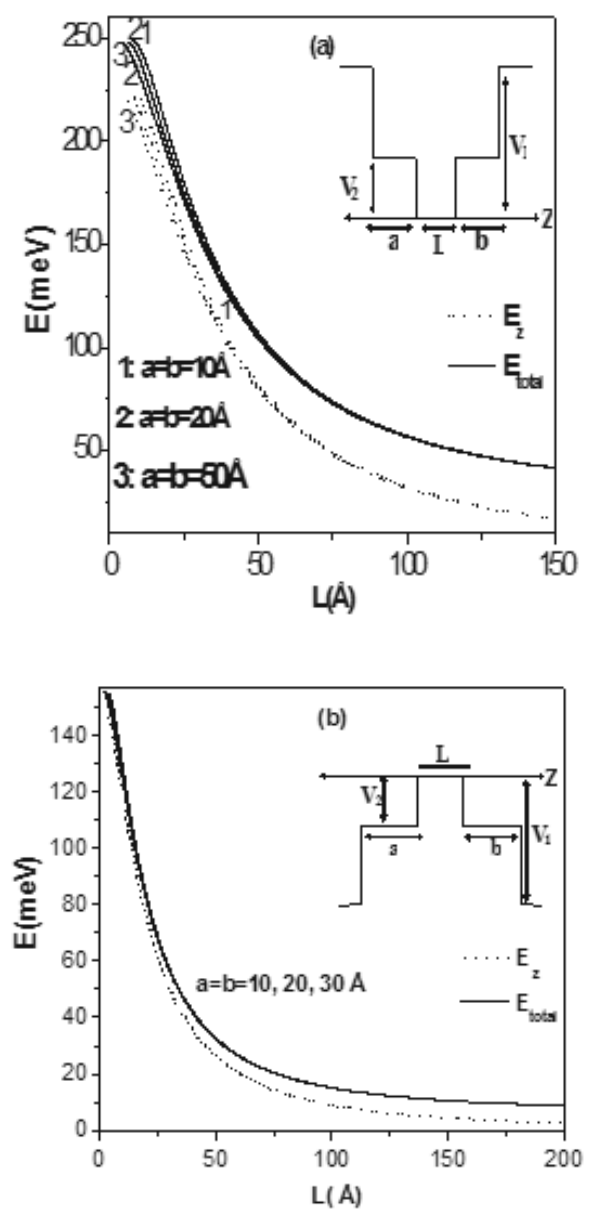

Figura 2. Energía del estado base en un punto cuántico de GaAs inmerso en un sistema de $\mathrm{Ga}_{0,6} \mathrm{Al}_{0,4} \mathrm{As}$ como función de la longitud del punto para diferentes espesores de las barreras: $\mathrm{a}=\mathrm{b}=10,20,50 \AA$.

Fuente: elaboración propia. 


\section{investigación |}

La línea sólida es la energía total, mientras que la línea punteada es la energía en la dirección $z$.

De la figura anterior se muestra que la energía del electrón (hueco) disminuye si la longitud del punto cuántico aumenta, además se observa que la energía para el confinamiento total (línea sólida) es mayor que cuando se considera el confinamiento $1 D$ (dirección $z$ ). Otro hecho fundamental está relacionado con la presencia de las barreras de potencial, se observa que su influencia es mayor para valores menores del ancho de las barreras con potencial de confinamiento $V_{2}$. Esta situación es similar tanto para las energías de electrones como para los huecos.

En la figura 3 presentamos la energía para el electrón en un punto cuántico de GaAs de radio $100 \AA$ inmerso en un sistema de $\mathrm{Ga}_{0,7} \mathrm{Al}_{0,3} \mathrm{As}$ como función de la posición del punto dentro del sistema $\mathrm{Ga}_{0,6} \mathrm{Al}_{0,4} \mathrm{As}$. Observamos que la energía disminuye a medida que el punto se acerca a la barrera de potencial $V_{1}$. Es importante recalcar que para pequeñas longitudes del punto cuántico $(\mathrm{L} \leq 10 \AA$ Å) la energía del estado fundamental presenta un comportamiento similar a la energía que presenta una impureza hidrogenoide en un pozo cuántico, alambre cuántico y punto cuántico.

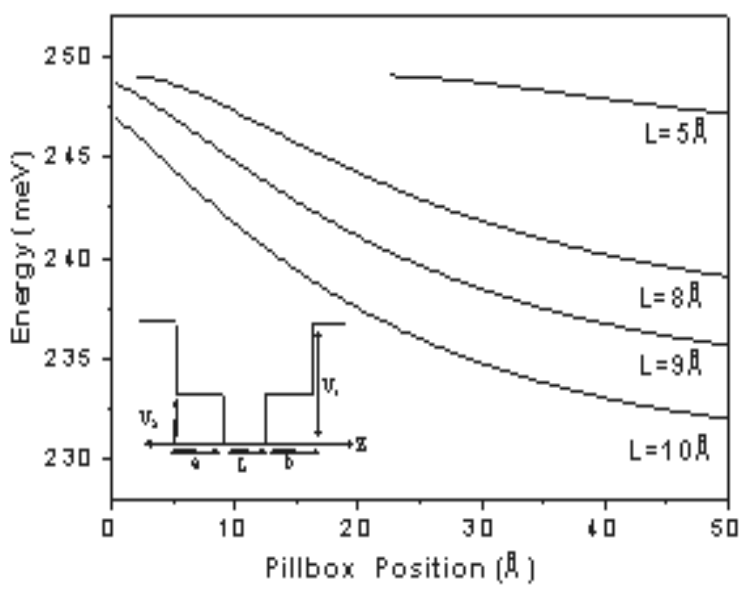

Figura 3. Energía del electrón en un pillbox de GaAs con $\mathrm{R}=100 \AA$ inmerso en un sistema de $\mathrm{Ga}_{0,6} \mathrm{Al}_{0,4} \mathrm{As}$ como función de la posición del pillbox dentro de la estructura de $\mathrm{Ga}_{0,6} \mathrm{Al}_{0,4} \mathrm{As}$, para diferentes longitudes.

Fuente: elaboración propia.
Para tener en cuenta los efectos de la presión y la temperatura sobre los estados energéticos de los portadores (electrón-hueco), el Hamiltoniano representado por las ecuaciones (1) y (4) puede ser escrito como:

$$
H=-\frac{\hbar^{2}}{2 m_{w, b}^{*}(P, T)} \nabla^{2}+V(z, P, T)
$$

En la ecuacion (16) los subíndices $w$ y $b$ representan los materiales del pozo cuántico y las barreras de las capas, respectivamente.

Las masas efectivas parabólicas de conducción $m_{w}^{*}(P, T)$ y $m_{b}^{*}(P, T)$ como función de la Presión (P) y Temperatura $(\mathrm{T})$ vienen determinados por la ecuación (17):

$m_{w c}(P, T)=\left[1+7, \Phi\left(\frac{2}{E_{g}(P, T)}+\frac{1}{E_{g}(P, T)+0,341}\right)\right]^{-1} m_{0}$.

Enlaecuación(17), $E_{g}$ es el gap de energía dependiente de la presión sobre el semiconductor de GaAs para bajas temperaturas en el punto $\Gamma$, el cual viene expresado así:

$E_{g}(P, T)=1,519+10,7 \times 10^{-3} P-5,405 \times 10^{-4} T^{2} /(T+204)$.

La dependencia de la masa efectiva de la barrera con la concentración de aluminio $x$ es:

$$
m_{b c}=m_{w c}+0,083 \times m_{0}
$$

De la ecuación (18) se debe tener en cuenta que cuando la temperatura y la presión hidrostática aplicada van a ser tomadas en cuenta, el potencial de confinamiento dependerá de estas cantidades físicas, esto es $V=V(z, P, T)$. Además tanto el ancho del 


\section{investigación}

pozo como el radio del punto cuántico dependen del esfuerzo aplicado, el cual puede ser determinado por su cambio fraccional, teniendo en cuenta que para una estructura Zinc-Blenda ese cambio es,

$$
\frac{\delta V}{V}=-3 P\left(S_{\mathbb{1}}+2 S_{\mathcal{1}}\right) \text {. }
$$

En la ecuación (20) los coeficientes $S_{11}\left(=1,16 \times 10^{-3} \mathrm{kbar}^{-}\right.$) y $S_{12}\left(=-3,7 \times 10^{-4} k b a \bar{r}\right)$ son las constantes elásticas para el GaAs.

A continuación presentamos los resultados de los efectos que tiene la presión hidrostática aplicada en el régimen de bajas temperaturas sobre nuestra estructura; para ello hemos utilizado los siguientes valores: $m_{e}^{*}=0,0665 m_{0}, m_{h}^{*}=0,3 m_{0}$ y el valor de la constante dieléctrica $\varepsilon=12,58$. Hemos asumido que la discontinuidad en el gap de $\mathrm{GaAs}-\mathrm{Ga} a_{1 \text {. }}$ ${ }_{x} A l_{x} A s$ del punto cuántico está distribuido cerca de $40 \%$ sobre la banda de valencia y a un $60 \%$ sobre la banda de conducción, con una diferencia en el gap $\Delta E_{g}$ entre GaAs y $G a_{1-x} A l_{x} A s$ dado como una función de la concentración de Aluminio para $x<0,45$, por $\Delta E_{g}(e V)=1,247 x$.

En la figura 4 se representa el comportamiento de la energía del electrón como función de la presión hidrostática, para dos valores del ancho del pozo, $\mathrm{L}=10 \AA$ (figura 4 (a)) y L=50 Å (figura 4 (b)). Se observa que estas energías decrecen con la presión hidrostática, principalmente debido a la disminución de la altura de la barrera $V_{2}$ con la presión. De acuerdo con estos resultados, un importante hecho es que la barrera de potencial $V_{2}$ modifica la energía de los portadores en los regímenes de confinamiento fuerte y débil de los portadores.
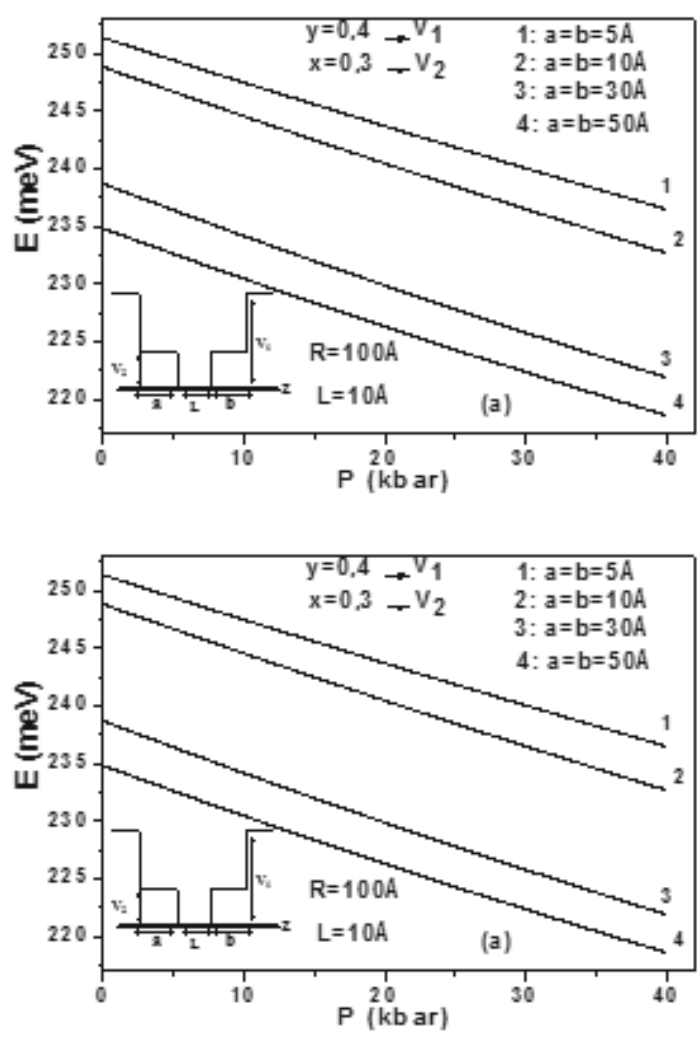

Figura 4. Energía del electrón como función de la presión hidrostática aplicada sobre un punto cuántico de GaAs- GaAs- $G a_{1-x} A l_{x} A s$ inmerso en un sistema de $G a_{1-y} A l_{y} A s$ con $x=0,3\left(V_{2}\right) y=0,4(V)$, para dos longitudes de (a) $L=10 \AA$ y (b) $L=50 \AA$.

Fuente: elaboración propia.

\section{CONCLUSIONES}

En este trabajo, usando la aproximación de la masa efectiva, se ha calculado la energía del estado fundamental del electrón y del hueco en un punto cuántico de GaAs-Ga $\mathrm{Al}_{1-\mathrm{x}} \mathrm{Al}_{\mathrm{x}} \mathrm{As}$, inmerso en una barrera de $\mathrm{Ga}_{1-\mathrm{y}} \mathrm{Al}_{\mathrm{y}} \mathrm{As}$ en función de la longitud del punto cuántico cuando el espesor de las barreras de potencial permanece constante, en función del espesor de la barrera de potencial para una longitud del punto cuántico fija y en función de la posición del punto cuántico dentro del material hospedero, $\mathrm{Ga}_{1-\mathrm{y}} \mathrm{Al}_{\mathrm{y}} \mathrm{As}$, para diferentes concentraciones $x$ e $y$ de aluminio. 


\section{investigación |}

Se encontró que las barreras de potencial del material hospedero, $\mathrm{Ga}_{1-\mathrm{y}} \mathrm{Al}_{\mathrm{y}} \mathrm{As}$, originan un aumento en la energía de los portadores (electrón y hueco) a medida que estas se aproximan al punto cuántico de GaAs$\mathrm{Ga}_{1-\mathrm{x}} \mathrm{Al}_{\mathrm{x}} \mathrm{As}$ y también que para alto confinamiento cuántico el sistema no presenta estados ligados.

Por otra parte, cuando se aplica presión hidrostática a la estructura, se encontró que la energía del estado fundamental del electrón decrece debido a la disminución de los potenciales de confinamiento $V_{1}$ y $V_{2}$.
Se comprobó que en puntos cuánticos, al igual que en pozos cuánticos, la energía de transición electrónhueco ligero $(e-l h)$ y electrón-hueco pesado $(e-h h)$ aumenta con la presión hidrostática. Se encontró una excelente concordancia con los resultados experimentales reportados en pozos cuánticos cuando se hizo el límite de nuestro punto cuántico a la estructura de pozo cuántico.

\section{REFERENCIAS}

[1] L. Esaki, and R. Tsu, "Superlattice and negative differential conductivity in semiconductors",IBM J. Res. Dev., vol. 14, pp. 20-21, May. 1987.

[2] X. Liu, X. Wang, and B. Gu,"Plasmons in asymmetric double quantum well structures", Eur. Phys. J. B., vol. 30, pp. 339342, Jul. 2002.

[3] Y. Fedutik, V. Temnov, O. Schops, and U. Woggon,"Exciton-plasmon-photon conversion in plasmonic nanostructures", Phys. Rev. B., vol.43, pp. 1368021-13680214, Sep. 2007.
[4] D. Chang, A. Sorensen, E. Demler, and M. Lukin, "A single-photon transistor using nanoscale surface plasmons", Nature, vol. 3, pp. 807-811, Aug. 2007.

[5] U. Hohenester, and J. Krenn, "Surface plasmon resonances of single and coupled metallic nanoparticles: A boundary integral method approach", Phys. Rev. B., vol.72, pp. 1954291-1954299, nov. 2005.

[6] S.Li, K. Chang, and J.Bai,"Effective-mass theory for hierarchical self-assembly of GaAs/ AlxGa1-xAs quantum dots",Phys. Rev. B., vol.71, pp. 1553011-1553017, Feb. 2005. 